\title{
A inserção dos terapeutas ocupacionais na rede de atenção à saúde do SUS em Minas Gerais entre os anos de 2005 e 2015
}

\author{
Melina Alves de Camargos, Marcela Vilela Barros Ferreira, Bruno Souza Bechara Maxta, \\ Alessandro Rodrigo Pedroso Tomasi
}

Departamento de Terapia Ocupacional, Universidade Federal de Minas Gerais - UFMG, Belo Horizonte, MG, Brasil.

\begin{abstract}
Resumo: Introdução: A terapia ocupacional é uma profissão envolvida nos campos da saúde, educação, cultura e assistência social. Estudos sobre o envolvimento do terapeuta ocupacional nas ações e nos serviços de saúde são fundamentais para a compreensão da sua participação no Sistema Único de Saúde (SUS). Objetivo: Identificar a inserção dos terapeutas ocupacionais atuantes na rede de atenção à saúde do SUS das macrorregiões de saúde (MRSs) do estado de Minas Gerais entre os anos de 2005 a 2015. Método: Foi realizada uma pesquisa documental de caráter descritivo. Foram utilizados documentos gerados pela base de dados do Cadastro Nacional de Estabelecimentos de Saúde no mês de julho de 2015. Ferramentas da estatística descritiva foram utilizadas para análise dos dados a partir das variáveis profissionais atuantes no SUS, tipos de estabelecimentos de saúde, macrorregiões de saúde e ano. Resultados: No período estudado, todas as MRSs apresentaram a inserção do terapeuta ocupacional. A Atenção Especializada, Atenção Primária e a Atenção Psicossocial foram identificadas como as redes de atenção à saúde predominantes de inserção profissional. A Urgência e Emergência, a Vigilância em Saúde e a Gestão em Saúde se apresentaram como potenciais campos de atuação profissional. Conclusão: A inserção dos terapeutas ocupacionais no SUS foi caracterizada por crescimento e presença em todas as MRSs em suas redes de atenção à saúde.
\end{abstract}

Palavras-chave: Sistema Único de Saúde, Recursos Humanos em Saúde, Terapia Ocupacional.

\section{The inclusion of occupational therapists in health care of the unified health system in minas gerais between the years 2005 and 2015}

\begin{abstract}
Introduction: Occupational therapy is a profession present in the fields of health, education, culture and social assistance. Studies addressing the role of occupational therapists in health services are essential for the understanding of their participation in the Unified Health System (SUS). Objective: To identify the inclusion of occupational therapists in the health care of the Minas Gerais macro-regions of SUS (MRS) in the years 2005-2015. Method: A descriptive documentary research was conducted. Documents generated by the data base of the National Registry of Health Establishments in July 2015 were used. Descriptive statistic tools were used for data analysis. Results: All MRS included occupational therapists. The Specialized Care, the Primary Care and the Psychosocial Care were identified as the health care fields where insertion of these professionals prevailed. The Urgency and Emergency, the Health Surveillance and the Health Management represented potential fields of action of this profession. Conclusion: The inclusion of occupational therapists in the SUS has been characterized by growth and presence in all MRS health care levels.
\end{abstract}

Keywords: Unified Health System, Health Manpower, Occupational Therapy.

\footnotetext{
Autor para correspondência: Bruno Souza Bechara Maxta, Escola de Educação Física, Fisioterapia e Terapia Ocupacional, Universidade Federal de Minas Gerais, Av. Pres. Antônio Carlos, 6627, Campus Pampulha, CEP 31270-901, Belo Horizonte, MG, Brasil, e-mail: brunobechara@ufmg.br Recebido em Ago. 17, 2016; 1ª Revisão em Nov. 17, 2016; Aceito em Dez. 19, 2016.
} 


\section{Introdução}

A terapia ocupacional é uma profissão que direciona os seus conhecimentos e açôes para os campos da saúde, educação, cultura e assistência social com intuito de promover a participação das pessoas em suas ocupaçôes. O profissional, ao oferecer oportunidades que permitam a participação em atividades significativas em casa, na escola, na comunidade e em outros settings assistenciais, contribui para qualificaçáo do cuidado e da assistência visando a promoção de saúde, prevenção de doenças e agravos e a reabilitação (AMERICAN..., 2015; KIELHOFNER, 2006; UNIVERSIDADE..., 2015).

No campo da saúde, o surgimento da profissão no Brasil se deu por meio de trabalhos voltados para as pessoas com deficiência e com transtornos mentais em instituiçóes totais asilares. Entre as décadas de 1950 e 1980, em virtude do reconhecimento da profissão no país, o terapeuta ocupacional foi inserido em diferentes serviços de reabilitação e tratamento de doenças mentais vinculados às estruturas previdenciárias de saúde. Neste período, a assistência à saúde brasileira era restritiva e excludente, ou seja, direcionada e focalizada para apenas um setor da população - os trabalhadores contribuintes e seus beneficiários. A parcela da população que ainda não tinha seus direitos sociais assegurados se amparava em serviços de saúde vinculados à filantropia e a restritas açôes de saúde pública no interior do país (MALFITANO; FERREIRA, 2011; SOARES, 1991).

No final da década de 1980, devido ao processo de redemocratização do Estado brasileiro para o setor da saúde, houve a possibilidade de participação popular nas discussóes nacionais sobre a reorientação das políticas de saúde vigentes no país (GIOVANELLA et al., 2013). A VIII Conferência Nacional de Saúde definiu as bases para a implementaçâo de um sistema de açôes e serviços que reconheceu a saúde como direito de todos brasileiros e responsabilidade do Estado, o que resultou na criação do Sistema Único de Saúde (SUS) (BRASIL, 1990).

\section{O SUS, como um}

[...] conjunto de açóes de serviços públicos de saúde, prestados por órgãos e instituiçôes públicas federais, estaduais e municipais, da administraçáo direta e indireta e das fundações mantidas pelo Poder Público (BRASIL, 1990, p. 1),

propóe um novo paradigma assistencial voltado às necessidades e demandas da população, considerando a pessoa como agente promotor de saúde (MALFITANO; FERREIRA, 2011). A universalidade, a integralidade, a equidade e o controle social são os princípios que direcionam os profissionais de saúde a superarem os desafios assistenciais por meio da valorizaçáo dos diferentes saberes em saúde e da implementação de novas práticas, que respondam aos reais problemas do cotidiano das pessoas (MALFITANO; FERREIRA, 2011; BRASIL, 1990).

Como forma de estabelecer um novo modelo assistencial de saúde no SUS, as Redes de Atenção à Saúde (RAS) conformam arranjos organizacionais, com intuito de interconectar e integrar os serviços de saúde presentes nos municípios e regióes de saúde de forma planejada e integral (MENDES, 2014). A Atenção Primária à Saúde (APS) se constitui como o centro de comunicação das RAS, além de ser reconhecida como o cenário de atenção à saúde de entrada preferencial do usuário para o SUS. A APS tem as suas açóes orientadas para as necessidades individuais e coletivas de cada território. Por meio destas espera-se um alto índice de resolutividade de problemas, atençáo horizontalizada e integral, responsabilizaçáo econômica e sanitária. Outro elemento constituinte da RAS são as unidades de serviços temáticos e/ou especializados educacionais, ambulatoriais, hospitalares, de vigilância em saúde, entre outros, que dáo apoio e suporte às açóes da APS. Além destas estruturas, os sistemas de apoio, logística e de governança têm o intuito de comunicar e interligar os pontos de atençáo à saúde (MENDES, 2014).

No processo de implementaçáo do SUS e de ampliação da sua assistência, novas ações e serviços foram criados como respostas ao cenário da saúde pública brasileira. A inserção do terapeuta ocupacional no SUS vem sendo viabilizada pela implementaçáo de seus programas e políticas. Atualmente, o terapeuta ocupacional é reconhecido como profissional potencial nos serviços da Rede de Atenção Psicossocial, Rede de Urgência e Emergência e, principalmente, na APS (CAMARGOS et al., 2014).

Diante do exposto, estudos que buscam identificar o envolvimento do terapeuta ocupacional nas açóes e serviços do SUS são fundamentais para a compreensão da trajetória histórico-política e da caracterizaçáo dos reais lugares de investimento dos profissionais. O objetivo do estudo foi identificar a inserção dos terapeutas ocupacionais atuantes nas diferentes açôes e serviços da rede de atenção à saúde do SUS nas macrorregióes de saúde do estado de Minas Gerais entre os anos de 2005 e 2015. É esperado que os resultados deste estudo colaborem para que os profissionais, organizados por meio de suas representaçôes associativas, sindicais e de categoria profissional, possam melhor exercer o papel de atores políticos para apresentação, defesa e ampliação do trabalho do terapeuta ocupacional no SUS. 


\section{Método}

Este estudo integra o projeto de pesquisa intitulado terapeutas ocupacionais no Sistema Único de Saúde em Minas Gerais desenvolvido pelo Departamento de Terapia Ocupacional da Universidade Federal de Minas Gerais.

Foi realizada uma pesquisa documental de caráter descritivo. A pesquisa documental tem como característica a utilizaçáo de documentos como fonte de coleta de dados, que podem ser caracterizados como fontes primárias, quando ainda não sofreram tratamento analítico, ou fontes secundárias, quando trabalhadas por algum tipo de análise (GIL, 2007).

Foram utilizados documentos gerados pela base de dados do Cadastro Nacional de Estabelecimentos de Saúde (CNES), por meio do sítio eletrônico do Departamento de Informática do Sistema Único de Saúde (DATASUS), no mês de julho de 2015. O CNES é uma base de dados pública que disponibiliza informaçôes de saúde (FAVERET, 2009). O DATASUS é responsável por gerir as informações e as ações de informatização no SUS com o intuito de envolver os estados e municípios no gerenciamento de dados sobre a saúde no país (BRASIL, 2015a).

Os dados coletados foram referentes ao número absoluto de terapeutas ocupacionais inseridos em serviços de atenção à saúde da administração direta, indireta e privada que atendem ao SUS do estado de Minas Gerais entre os anos de 2005 e 2015. As variáveis Macrorregião de Saúde (MRS), tipo de estabelecimento de saúde, e número de terapeutas ocupacionais que atendem o SUS foram identificadas, organizadas e descritas por meio de mapa e tabela de referência, tabelas de contingência e gráfico de frequências.

Como procedimentos de coleta de dados, foram utilizados os seguintes menus no sítio eletrônico do DATASUS, na sequência indicada: Informações de Saúde (TABNET); Rede Assistencial; CNES/Recursos Humanos até julho de 2007 - Ocupaçôes classificadas pela CBO 1994 e CNES/Recursos Humanos a partir de agosto de 2007 - Ocupaçôes classificadas pela CBO 2002. A utilização dos dois últimos menus é justificada pelo período selecionado no estudo. Na sequência, foi selecionada a opção "Ocupaçôes" e, depois, determinada a abrangência geográfica (com seleção por mapa ou lista), sendo escolhida a opção "Minas Gerais". Os dados selecionados corresponderam à correlação entre as opçóes "macrorregião de saúde/município" (linha), "tipo de estabelecimento” (coluna), "atende o sus” (conteúdo) e "terapeuta ocupacional" (ocupaçóes em geral). Neste processo foram marcadas as opçóes de exibição de linhas zeradas para que os municípios que náo contivessem terapeutas ocupacionais aparecessem na busca. Os dados foram gerados por tabelas automáticas do sítio eletrônico do DATASUS no formato "COPIA COMO.CSV", armazenados em planilhas eletrônicas e arquivados em pastas virtuais individualizadas. Foram confeccionadas tabelas no software livre Open Office, para edição de dados e geração do formato de arquivo específico para leitura no software de geoprocessamento QuantumGIS-versão 2.2.

Ferramentas da estatística descritiva foram utilizadas para a análise dos dados (TORRES; REIS; PAGANO, 2015). Mapas de referência foram utilizados para organizar e descrever a relação entre as variáveis macrorregião de saúde e terapeutas ocupacionais atuantes no SUS nos anos inicial e final do estudo. Uma tabela de referência foi utilizada para identificar e descrever os serviços de saúde que apresentaram terapeutas ocupacionais atuantes no SUS em consideração com a terminologia proposta pela Relação Nacional de Açóes e Serviços de Saúde (RENASES). A RENASES é um documento do Ministério da Saúde que tem como pretensão agrupar e classificar todas as açóes e serviços de saúde oferecidos pelo SUS independente da sua natureza administrativa, tanto da administraçáo direta quanto da indireta, contemplando assim, todos os serviços assistenciais e açôes em saúde no país (BRASIL, 2012). Esta classificação propóe a divisão das açôes e dos serviços de saúde em cinco componentes, sendo eles: APS, Urgência e Emergência; Atenção Psicossocial, Atenção Especializada (AE) e Vigilância em Saúde. Devido à inexistência da categoria "Gestão em Saúde", foi necessária a criação da mesma para alocação de seu serviço de saúde. Tabelas de contingência foram utilizadas para descrever a relação das frequências absolutas dos terapeutas ocupacionais atuantes no SUS em seus pontos de atenção à saúde, nas ações e serviços de saúde e nas MRSs nos anos inicial e final do estudo. Um gráfico de frequência foi utilizado para a exibiçâo gráfica dos dados dos terapeutas ocupacionais atuantes no SUS. A medida de variaçáo foi utilizada para representar a distribuição dos terapeutas ocupacionais atuantes no SUS do estado de Minas Gerais nos últimos onze anos.

\section{Resultados e Discussão}

Minas Gerais é um estado brasileiro localizado na região Sudeste do país que apresentou, em 2010, população estimada em 19.597.530 habitantes 
distribuída em 853 municípios (INSTITUTO..., 2010). O Plano Diretor de Regionalização da Saúde de Minas Gerais (PDR/MG) organiza os municípios do estado em 13 Macrorregióes, que considera as possibilidades de oferta de acesso aos serviços intermunicipais de saúde de maior complexidade tecnológica e 76 Microrregióes de Saúde, que prevê a oferta de serviços de atenção primária e ambulatoriais e hospitalares de média complexidade tecnológica para açóes intermunicipais. Por meio das RAS são definidas e priorizadas as alocações dos recursos loco-regionais e as açôes para a descentralização programática da assistência à saúde do estado por meio de diferentes serviços (MALACHIAS; LELES; PINTO, 2010).

Os terapeutas ocupacionais foram apresentados em todas as MRS, em diferentes serviços de saúde em todos os pontos da rede de atençáo do SUS de Minas Gerais.

Em 2005, todas as MRSs apresentaram pelo menos um terapeuta ocupacional. A MRS Centro foi a que apresentou o maior número de profissionais (290), seguida pela MRS Sul (75) (Figura 1). As MRSs Jequitinhonha (01) e Nordeste (05) apresentaram o menor número de profissionais. A distribuição dos terapeutas ocupacionais no estado, em 2015, mostrou-se similar quanto ao gradiente de concentração dos profissionais em 2005 (Figura 2). No que diz respeito à variação do número de terapeutas ocupacionais, houve um aumento de, aproximadamente, 124\% nas 13 MRSs. Destacaram-se a MRS Jequitinhonha, com aumento de $1700 \%$, e a MRS Nordeste, com o aumento de $280 \%$ no número de profissionais.
Rodrigues (2008), em estudo sobre a distribuição de profissionais médicos em Minas Gerais, concluiu que, nesta categoria profissional, as maiores concentraçóes de profissionais foram identificadas nas regiōes do estado reconhecidas como pólos tradicionais de formação e grandes centros urbanos, como as MRSs Sul e Centro. Os resultados do presente estudo sugerem que a distribuição dos terapeutas ocupacionais de Minas Gerais pode indicar fenômeno semelhante ao dos profissionais médicos.

Sobre a inserção do terapeuta ocupacional, a Atenção Especializada contou com o maior número absoluto de profissionais do ano inicial ao ano final do estudo e com a quarta maior taxa de variação (43\%), como exposto na Tabela 1. A MRS que mais envolveu terapeutas ocupacionais nesta rede assistencial foi a Jequitinhonha (1300\%), seguida pela Leste do Sul (325\%). As MRSs Triângulo do Norte (-24\%), Nordeste (-18\%) e Noroeste (-18\%) apresentaram declínio no número de profissionais.

Quanto às açôes e serviços de saúde, notou-se que o hospital geral apresentou a maior taxa de variaçáo profissional (222\%), como evidenciado na Tabela 2. O contexto hospitalar ainda representa um importante serviço de acesso ao SUS. Contudo, com a mudança de paradigma no modelo assistencial, este campo passou a ser visto como estação do cuidado, e não mais exclusivamente como o principal eixo do setor saúde (CECÍLIO; MEHRY, 2013). A prática do terapeuta ocupacional acompanhou as transformaçóes ocorridas neste cenário e, atualmente, o profissional conduz sua prática para ao tratamento e reabilitação de casos agudos com foco na funcionalidade e

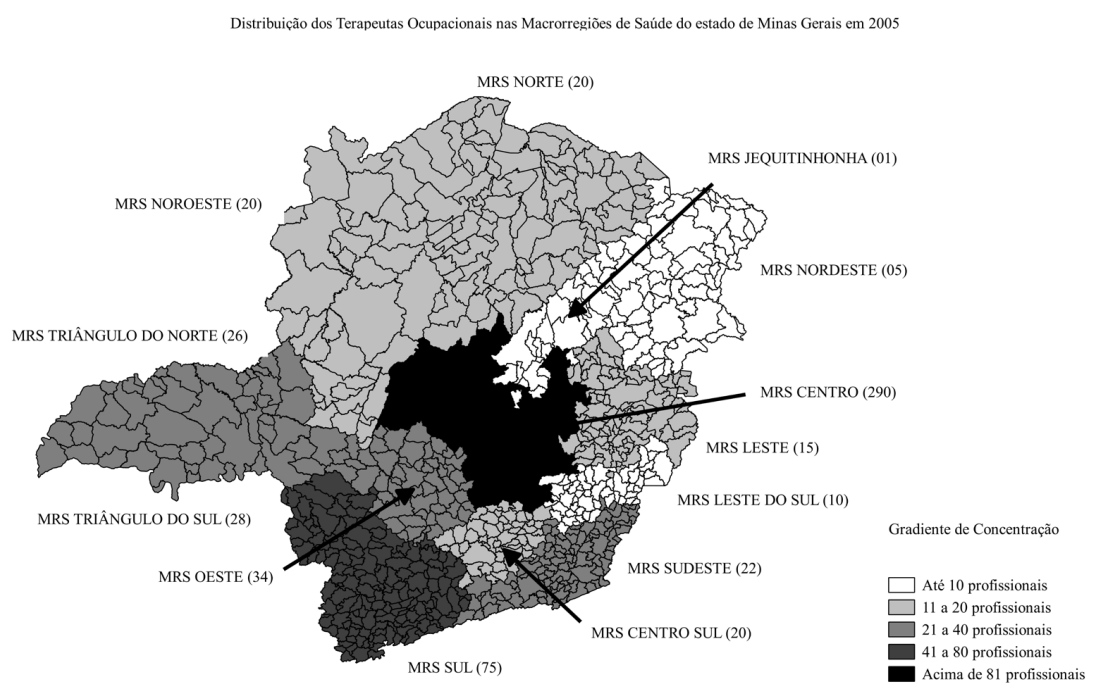

Figura 1. Distribuição dos terapeutas ocupacionais nas Macrorregiões de Saúde do estado de Minas Gerais em 2005. Fonte: Brasil (2015b). 


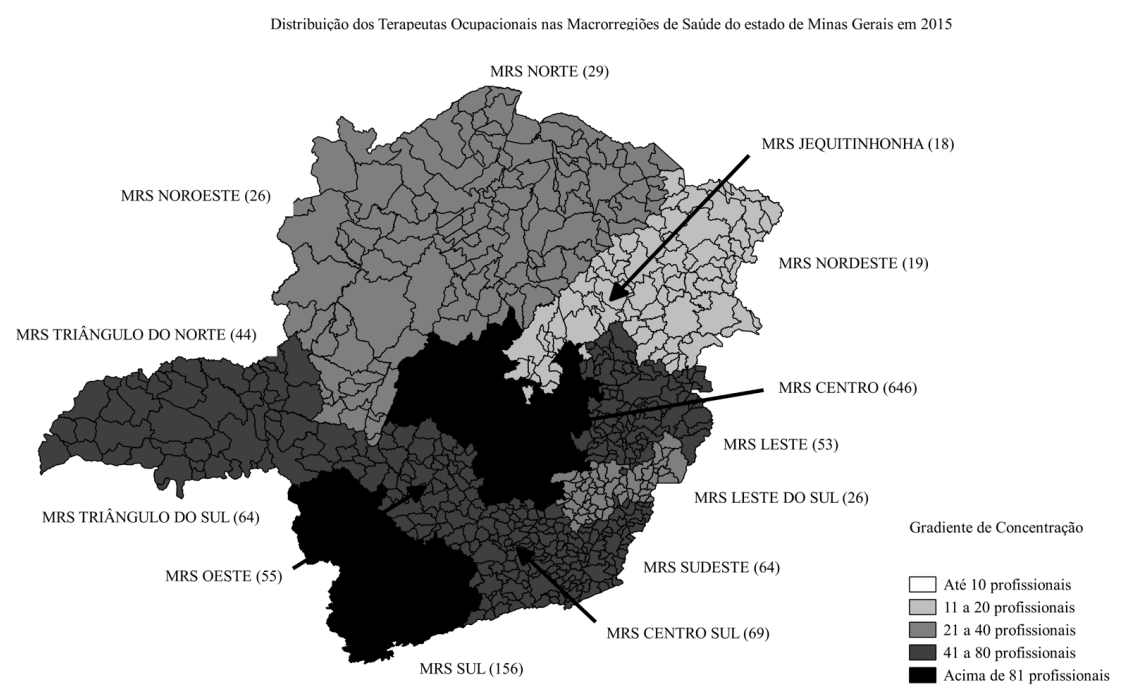

Figura 2. Distribuição dos terapeutas ocupacionais nas Macrorregiões de Saúde do estado de Minas Gerais em 2015. Fonte: Brasil (2015b).

desempenho ocupacional dos indivíduos (BORGES; LEONI; COUTINO, 2012; GALHEIGO, 2008).

A APS apresentou a mesma tendência de crescimento profissional, com ascensão a partir de 2008 e decréscimo no último ano (Figura 3), apresentando a segunda maior taxa de crescimento (268\%). A MRS que apresentou maior variação de terapeutas ocupacionais foi a Sudeste (1500\%), seguida pela MRS Leste (950\%). Foi possível identificar que a única MRS que obteve declínio no quadro de profissionais foi a Oeste (-29\%) (Tabela 2).

O Centro de Saúde e o Posto de Saúde revelaram-se como os estabelecimentos de saúde semelhantes (BRASIL, 2016) que mais inseriram profissionais na APS (Tabela 2). Tal crescimento pode ser justificado pela criação do Núcleo de Apoio à Saúde da Família, que tem o intuito de apoiar as Equipes de Saúde da Família, a fim de ampliar o escopo das açóes na APS, bem como contribuir para o índice de resolutividade de problemas de saúde territoriais (BRASIL, 2011). As açôes terapêuticas ocupacionais na APS direcionam-se para o desempenho ocupacional da pessoa em seu contexto familiar e comunitário, potencializando a autonomia e a participação social (ROCHA; SOUZA, 2011).

Os serviços da Atenção Psicossocial, a partir de seu reconhecimento no DATASUS, em 2009, apresentou inserção e crescimento de terapeutas ocupacionais no período estudado (Figura 3). Não foi possível identificar a variação do número de profissionais nesta rede assistencial, devido à tardia identificação dos profissionais em seus serviços (Tabela 2). A Lei 10.216/2001, que dispóe sobre a proteção e os direitos das pessoas em sofrimento mental e redireciona o modelo assistencial em saúde mental, foi o marco legislativo que preconiza cuidados humanizados, menos invasivos, e serviços abertos de base territorial (BRASIL, 2001). A Portaria no 336/2002 estabelece os Centros de Atenção Psicossocial (CAPS) em suas diferentes modalidades (álcool e outras drogas e infantil), como componentes que foram incorporados na atual Rede de Atenção Psicossocial (BRASIL, 2002).

Nestes serviços, cabe ao terapeuta ocupacional integrar suas ações aos princípios da Reabilitação Psicossocial e extrapolar as suas intervençôes para settings não tradicionais nos territórios das pessoas, viabilizando trocas sociais. É desafio para a terapia ocupacional direcionar as suas práticas para açôes que potencializem o desempenho ocupacional das pessoas em seus projetos de vida (COSTA; ALMEIDA; ASSIS, 2015).

Nos serviços de Urgência e Emergência, foi observado considerável crescimento de profissionais de 2005 a 2009, seguidos por períodos oscilatórios e com significativo declínio a partir de 2014 (Figura 3). Esta rede apresentou a maior taxa de crescimento (400\%), porém ressalta-se que este valor não representa um número expressivo de terapeutas ocupacionais. A MRS Centro apresentou variação de 200\% (Tabela 2).

Nesta, o Pronto Socorro foi o serviço que mais inseriu profissionais. Este tipo de serviço apresentou taxa de crescimento de $100 \%$ (Tabela 2). Não foi possível caracterizar as ações terapêuticas ocupacionais neste serviço, pois não foram identificados estudos 


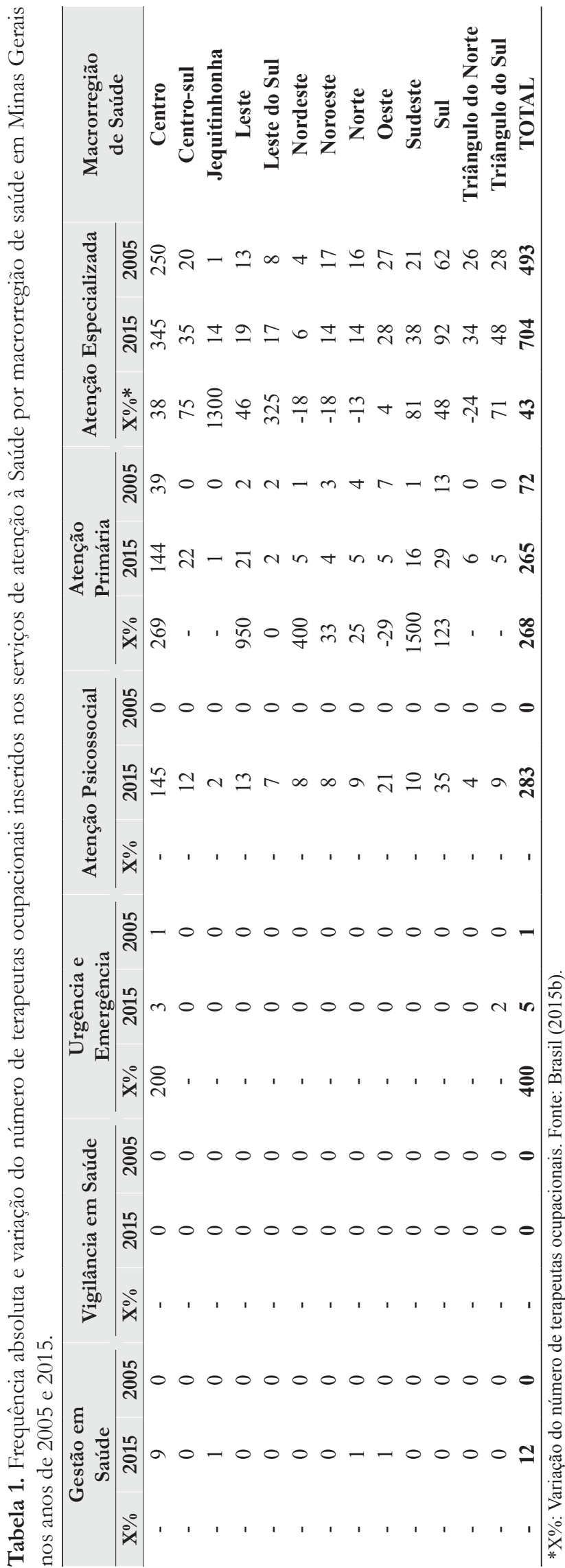


Tabela 2. Variação do número de terapeutas ocupacionais inseridos nos serviços de saúde por rede de atenção à saúde nos anos de 2005 e 2015.

\begin{tabular}{|c|c|c|c|c|}
\hline \multirow{2}{*}{$\begin{array}{c}\text { Variação } \\
(\%)\end{array}$} & \multicolumn{2}{|c|}{ Ano } & \multirow{2}{*}{ Serviços de Saúde } & \multirow{2}{*}{ Redes de Atenção à Saúde } \\
\hline & 2015 & 2005 & & \\
\hline 39 & 399 & 288 & Clínica Especializada & \multirow{9}{*}{ Atenção Especializada } \\
\hline 0 & 7 & 7 & Consultório & \\
\hline- & 0 & 0 & Cooperativa & \\
\hline-15 & 60 & 71 & Hospital Especializado & \\
\hline 222 & 174 & 54 & Hospital Geral & \\
\hline - & 1 & 0 & Hospital Dia & \\
\hline 11 & 42 & 38 & Policlínica & \\
\hline-43 & 20 & 35 & $\begin{array}{l}\text { Unidade de Serviço de Apoio de } \\
\text { Diagnose e Terapia }\end{array}$ & \\
\hline- & 1 & 0 & $\begin{array}{l}\text { Central de Notificação, Captação e } \\
\text { Distribuição de Órgãos Estadual }\end{array}$ & \\
\hline- & 2 & 0 & Academia da Cidade & \multirow{7}{*}{ Atenção Primária } \\
\hline- & 1 & 0 & Centro de Apoio à Saúde da Família & \\
\hline 259 & 255 & 71 & Centro de Saúde* & \\
\hline 200 & 3 & 1 & Posto de Saúde** & \\
\hline- & 1 & 0 & $\begin{array}{l}\text { Unidade de Atenção em Regime } \\
\text { Residencial }\end{array}$ & \\
\hline- & 2 & 0 & Unidade Mista & \\
\hline- & 1 & 0 & Telessaúde & \\
\hline- & 283 & 0 & Centro de Atenção Psicossocial & Atenção Psicossocial \\
\hline- & 0 & 0 & Centro de Atenção Hemoterápica & \multirow{4}{*}{ Urgência e Emergência } \\
\hline- & 2 & 0 & Central de Regulação de Serviços & \\
\hline - & 1 & 0 & Pronto Atendimento & \\
\hline 100 & 2 & 1 & Pronto Socorro & \\
\hline- & 0 & 0 & Unidade de Vigilância em Saúde & Vigilância em Saúde \\
\hline- & 12 & 0 & Secretaria de Saúde & Gestão em Saúde \\
\hline
\end{tabular}

*Centro de Saúde: Unidade para realização de atendimentos de atenção básica e integral a uma população, de forma programada ou não, nas especialidades básicas, podendo oferecer assistência odontológica e de outros profissionais de nível superior. A assistência deve ser permanente e prestada por médico generalista ou especialista nestas áreas. Podendo ou não oferecer: SADT e Pronto atendimento 24 Horas; **Posto de Saúde: Unidade destinada à prestação de assistência a uma determinada população, de forma programada ou não, por profissional de nível médio, com a presença intermitente ou não do profissional médico. Fonte: Relação Nacional de Ações e Serviços de Saúde (Brasil, 2015b).

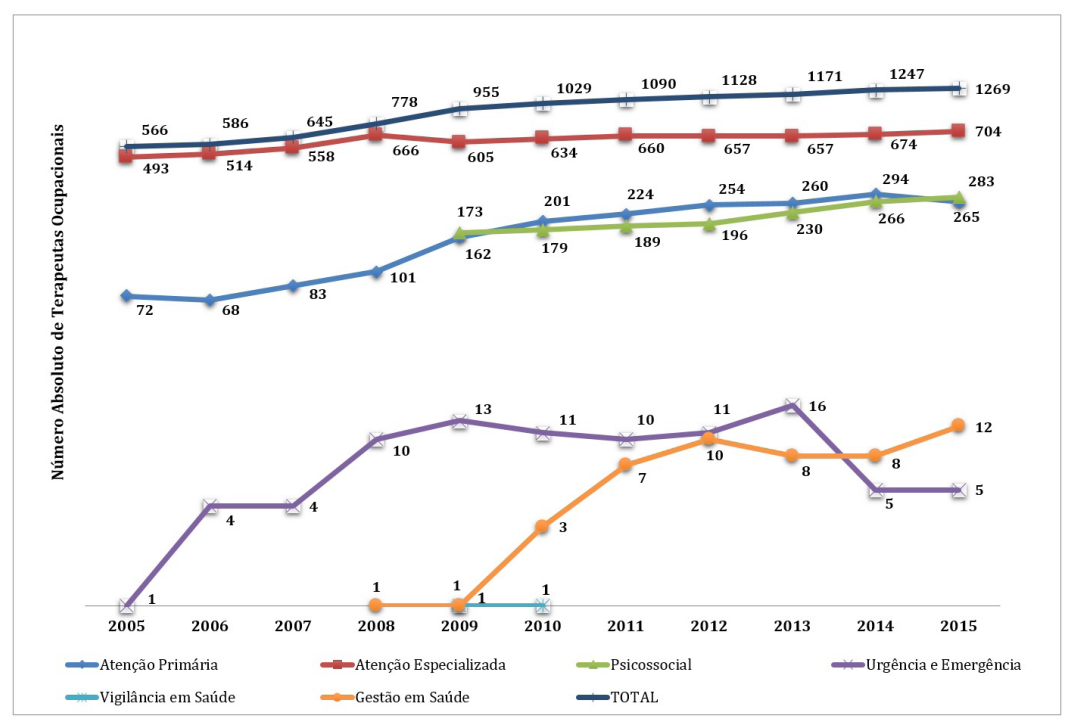

Figura 3. Frequência absoluta de terapeutas ocupacionais nos serviços de atenção à saúde em Minas Gerais entre os anos de 2005 e 2015. Fonte: Brasil (2015b). 
brasileiros que correlacionem a atuação profissional com os princípios do SUS. Ao considerar a notável presença do terapeuta ocupacional neste âmbito, destaca-se a importância do engajamento do mesmo em produçôes técnico-científicas.

Quanto à rede da Vigilância em Saúde, verificou-se a presença de um profissional nos anos de 2009 e 2010, o que sugere que historicamente este não é um campo tradicional da terapia ocupacional no estado (Figura 3). A inserção dos profissionais nesta rede assistencial pode ser viabilizada por práticas interdisciplinares dos serviços de Vigilância em Saúde do Trabalhador, representados pelos Centros de Referência em Saúde do Trabalhador, entre outros que respondam às questóes de saúde e trabalho no SUS (Tabela 2). Nestes locais, o terapeuta ocupacional objetiva fiscalizar, desenvolver intervenções educativas e reabilitadoras, sensibilizar trabalhadores e contratantes quanto aos riscos e adoecimentos relacionados ao trabalho, bem como mobilizar açôes transformadoras em relação ao processo de trabalho em uma abordagem articulada e intersetorial (DALDON; LANCMAN, 2013; VAZ, 2015).

$\mathrm{Na}$ Gestão em Saúde, o envolvimento do profissional se deu a partir de 2008, com crescimento gradativo a partir de 2009, declínio em 2013 e ascensão em 2015 (Figura 3). Este cenário, como outros que não prestam assistência direta à saúde, durante muitos anos, não foi campo de investimento profissional. Nesse campo, é indicado para o terapeuta ocupacional gestor buscar conhecimentos sobre o sistema de saúde e sua operacionalidade, de forma a viabilizar os recursos para atingir os objetivos das ações e serviços que ele coordena (AMERICAN..., 2015). Deste modo, cabe ao terapeuta ocupacional aplicar os conhecimentos teóricos e práticos do profissional sobre a ocupação, territorialidade, contextos sociais, determinantes de saúde e sobre o próprio sistema para qualificar a gestão em saúde (CRUZ; SOUZA; EMMEL, 2014; CARLO et al., 2009).

\section{Conclusão}

Em Minas Gerais, a inserção dos terapeutas ocupacionais no SUS, no período de 2005 a 2015, foi caracterizada por significativo crescimento, com a presença do profissional em todas as redes de atenção à saúde e em todas as MRSs do estado. No entanto, a inserção ainda se concentra em serviços que prestam assistência de cuidado direta à saúde nas MRSs Centro e Sul. Deste modo, as ações e serviços vinculados às redes de Vigilância em Saúde e Gestão em Saúde representam novos campos de investimentos propícios para o terapeuta ocupacional.

Os resultados deste estudo colaboram na qualificação das informações sobre o terapeuta ocupacional no estado. Sugerem-se novos estudos que objetivam compreender a distribuição e a mobilidade destes profissionais, de forma a subsidiar informações que podem qualificar a formulação e a implementação de políticas públicas de inserção e de formação do terapeuta ocupacional para o trabalho em Minas Gerais.

\section{Referências}

AMERICAN OCCUPATIONAL THERAPY ASSOCIATION - AOTA. Estrutura da prática da Terapia Ocupacional: Domínio e Processo. Revista de Terapia Ocupacional da Universidade de São Paulo, São Paulo, v. 26, p. 1-49, 2015. Número especial.

BORGES, F.; LEONI, T. F.; COUTINO, I. Terapia Ocupacional no contexto hospitalar: um delineamento da profissão em hospitais gerais e especializados na cidade de Salvador, BA. Cadernos de Terapia Ocupacional da UFSCar, São Carlos, v. 20, n. 3, p. 425-433, 2012.

BRASIL. Lei no 8.080, de 19 de setembro de 1990. Dispóe sobre as condiçốes para a promoção, proteção e recuperação da saúde, a organização e o funcionamento e dá outras providências. Diário Oficial da Uniāo, Brasília, DF, 20 set. 1990. Disponível em: <http://www.planalto. gov.br/ccivil_03/Leis/L8080.htm>. Acesso em: 1 nov. 2015.

BRASIL. Lei no 10.216, de 6 de junho de 2001. Dispóe sobre a proteçáo e os direitos das pessoas portadoras de transtornos mentais e redireciona o modelo assistencial em saúde mental. Diário Oficial da República Federativa do Brasil, Brasília, DF, 6 abr. 2001. Disponível em: <http://www.planalto.gov.br/ccivil_03/leis/leis_2001/ 110216.htm>. Acesso em: 1 set. 2015.

BRASIL. Portaria GM no 336, de 19 de fevereiro de 2002. Dispóe sobre CAPS I, CAPS II, CAPS III, CAPS i II e CAPS ad II. Diário Oficial da União, Brasília, DF, 19 fev. 2002. Disponível em: <http://dtr2004.saude. gov.br/susdeaz/legislacao/arquivo/39_Portaria_336_ de_19_02_2002.pdf>. Acesso em: 1 nov. 2015.

BRASIL. Ministério da Saúde. Gabinete do Ministro. Portaria ${ }^{\circ} 2.488$ de 21 de outubro de 2011. Aprova a Política Nacional de Atenção Básica, estabelecendo a revisão de diretrizes e normas para a organização da Atenção Básica, para a Estratégia Saúde da Família (ESF) e o Programa de Agentes Comunitários de Saúde (PACS). Diário Oficial da União, Brasília, DF, 21 out. 2011. Disponível em: <http://bvsms.saude.gov.br/bvs/saudelegis/ gm/2011/prt2488_21_10_2011.html>. Acesso em: 5 dez. 2016 .

BRASIL. Ministério da Saúde. Relação Nacional de Ações e Serviços de Saúde. Brasília, 2012. Disponível em: 
$<$ http://conitec.gov.br/images/Protocolos/Renases2012. pdf>. Acesso em: 1 nov. 2015.

BRASIL. Ministério da Saúde. Relatório DATASUS 20112014. Brasília, 2015a. Disponível em: <http://datasus. saude.gov.br/images/RelatorioExecutivodaGestao/RelatorioExecutivodaGestao2011-2014.pdf>. Acesso em: 1 nov. 2015 .

BRASIL. Ministério da Saúde. Departamento de Informática do Sistema Único de Saúde. Cadastro Nacional dos Estabelecimentos de Saúde do Brasil - CNES. Brasília, 2015b.

BRASIL. Ministério da Saúde. Informaçôes de Saúde. Tipos de estabelecimento. Brasília, 2016. Disponível em: <http://tabnet.datasus.gov.br/cgi/cnes/tipo_estabelecimento.htm>. Acesso em: 5 dez. 2016.

CAMARGOS, M. A. et al. Terapia Ocupacional nas Redes de Atenção do Sistema Único de Saúde. In: JORNADA ACADÊMICA DE TERAPIA OCUPACIONAL, 1., 2014, Belo Horizonte. Anais... Belo Horizonte: UFMG, 2014. Disponível em: <http://media.wix.com/ ugd/810b1e_77f027d607ae4390b8eeffe497c2bc9f. pdf>. Acesso em: 18 nov. 2015.

CARLO, M. M. R. P. et al. Planejamento e gerenciamento de serviços e conteúdos de formação profissional em terapia ocupacional. Interface - Comunicação, Saúde, Educação, Botucatu, v. 13, n. 29, p. 445-453, 2009. http:// dx.doi.org/10.1590/S1414-32832009000200016.

CECÍLIO, L. C. O.; MEHRY, E. E. A integralidade do cuidado com eixo da gestão hospitalar. Campinas: [s.n.], 2013. Disponível em: <http://www.hc.ufmg.br/gids/Integralidade.pdf>. Acesso em: 16 out. 2015.

COSTA, L. A.; ALMEIDA, S. C.; ASSIS, M. G. Reflexôes epistêmicas sobre a Terapia Ocupacional no campo da Saúde Mental. Caderno de Terapia Ocupacional UFSCar, São Carlos, v. 23, n. 1, p. 189-196, 2015. http:// dx.doi.org/10.4322/0104-4931.ctoARL432.

CRUZ, D. M. C.; SOUZA, F.; EMMEL, M. L. G. Formação do terapeuta ocupacional para a gestão. Revista de Terapia Ocupacional da Universidade de São Paulo, Sáo Paulo, v. 25, n. 3, p. 309-316, 2014. http://dx.doi. org/10.11606/issn.2238-6149.v25i3p309-316.

DALDON, M. T.; LANCMAN, S. Vigilância em saúde do trabalhador. Revista Brasileira de Saúde Ocupacional, São Paulo, v. 38, n. 127, p. 92-106, 2013. http://dx.doi. org/10.1590/S0303-76572013000100012.

FAVERET, A. C. S. C. (Ed.). Prontuários de bases de dados: informação sistematizada para as contas de saúde do Brasil. Brasília: IPEA, 2009.

GALHEIGO, S. M. Terapia ocupacional, a produção do cuidado em saúde e o lugar do hospital: reflexôes sobre a constituição de um campo de saber e prática. Revista de Terapia Ocupacional da Universidade de São Paulo, São Paulo, v. 19, n. 1, p. 20-28, 2008.
GIL, A. C. Como elaborar projetos de pesquisa. São Paulo: Atlas, 2007.

GIOVANELLA, L. et al. (Ed.). Politicas e Sistema de Saúde no Brasil. Rio de Janeiro: Editora Fio Cruz, 2013.

INSTITUTO BRASILEIRO DE GEOGRAFIA E ESTATÍSTICA - IBGE. Estados@: Minas Gerais. Rio de Janeiro, 2010. Disponível em: <http://www.ibge.gov. br/estadosat/perfil.php?sigla=mg $>$. Acesso em: 16 nov. 2015.

KIELHOFNER, G. El paradigma contemporáneo: un retorno a lao cupación como el centro de la profesión. In: KIELHOFNER, G. Fundamentos conceptuales de la terapia ocupacional. Buenos Aires: Médica Panamericana, 2006. p. 64-71.

MALACHIAS, I.; LELES, F. A. G.; PINTO, M. A. S. Plano Diretor de Regionalização da Saúde de Minas Gerais. Belo Horizonte: Secretaria de Estado de Saúde de Minas Gerais, 2010.

MALFITANO, A. P. S.; FERREIRA, A. P. Saúde pública e terapia ocupacional: apontamentos sobre relações históricas e atuais. Revista de Terapia Ocupacional da Universidade de São Paulo, São Paulo, v. 22, n. 2, p. 102-109, 2011.

MENDES, E. V. Comentários sobre as redes de atenção à Saúde no SUS. Divulgação em Saúde para Debate, Rio de Janeiro, n. 52, p. 38-49, 2014.

ROCHA, E. F; SOUZA, C. C. B. X. Terapia Ocupacional em reabilitação na Atenção Primária à Saúde: possibilidades e desafios. Revista de Terapia Ocupacional da Universidade de São Paulo, São Paulo, v. 22, n. 1, p. 36-44, 2011.

RODRIGUES, F. G. Médicos em Minas Gerais: projeções para o período 2010-2020. 2008. 104 f. Dissertação (Mestrado em Demografia) - Universidade Federal de Minas Gerais, Belo Horizonte, 2008.

SOARES, L. B. T. Terapia Ocupacional: lógica do capital ou do trabalho? São Paulo: Hucitec, 1991.

TORRES, H. C.; REIS, I. F; PAGANO, A. S. (Ed.) Emponderamento do pesquisador nas ciências da saúde. Belo Horizonte: FALE/UFMG, 2015.

UNIVERSIDADE DE SÃO PAULO - USP. Faculdade de Medicina de Ribeirão Preto - FMRPUSP. Terapia Ocupacional. Ribeirão Preto, 2015. Disponível em: <http://www.fmrp.usp.br/site-graduacao/graduacao/ cursos-oferecidos-pela-fmrp/terapia-ocupacional/>. Acesso em: 1 jul. 2015.

VAZ, R. S. C. A atuação da terapia ocupacional no centro de referência em saúde do trabalhador: uma revisão bibliográfica (2007 a 2014). 2015. 38 f. Monografia (Trabalho de Conclusão de Curso em Terapia Ocupacional) - Universidade de Brasília, Brasília, 2015. 


\section{Contribuição dos Autores}

Bruno Souza Bechara Maxta: orientação da pesquisa, construção do referencial teórico, análise dos dados e revisão do texto. Alessandro Rodrigo Pedroso Tomasi: orientação da pesquisa; construção da metodologia e análise dos dados; revisão do texto. Melina Alves de Camargos: construção do referencial teórico, construção da metodologia, coleta, análise e apresentação dos dados; revisão do texto. Marcela Vilela Barros Ferreira: construção do referencial teórico; coleta, análise e apresentação dos dados; revisão do texto. Todos os autores aprovaram a versão final do texto. 